\title{
Minor grove binding ligands disrupt PARP-1 activation pathways
}

\author{
Kirill I. Kirsanov ${ }^{1, *}$, Elena Kotova ${ }^{2, *}$, Petr Makhov ${ }^{2}$, Konstantin Golovine ${ }^{2}$, Ekaterina \\ A. Lesovaya ${ }^{1}$, Vladimir M. Kolenko ${ }^{2}$, Marianna G. Yakubovskaya ${ }^{1}$, Alexei V. Tulin ${ }^{2}$ \\ 1 Blokhin Cancer Research Center RAMS, Moscow, Russia \\ ${ }^{2}$ Fox Chase Cancer Center, Philadelphia, PA \\ * These authors contributed equally to this work \\ Correspondence to: Alexei V. Tulin, email: Alexei.Tulin@fccc.edu \\ Keywords: PARP-1, PARP-1 inhibitors, DNA-binding small molecules, poly(ADP-ribose) \\ Received: December 25, 2013 Accepted: January 3, $2014 \quad$ Published: January 3, 2014
}

This is an open-access article distributed under the terms of the Creative Commons Attribution License, which permits unrestricted use, distribution, and reproduction in any medium, provided the original author and source are credited.

\section{ABSTRACT:}

PARP-1 is a nuclear enzyme regulating transcription, chromatin restructuring, and DNA repair. PARP-1 is activated by interaction with NAD+, DNA, and core histones. Each route of PARP-1 activation leads to somewhat different outcomes. PARP1 interactions with core histones control PARP-1 functions during transcriptional activation in euchromatin. DNA-dependent regulation of PARP-1 determines its localization in heterochromatin and PARP-1-dependent silencing. Here we address the biological significance of DNA-dependent PARP-1 regulation in vitro and in vivo. We report that minor grove binding ligands (MGBLs) specifically target PARP-1 interaction with DNA, and, hence, the DNA-dependent pathway of PARP-1 activation. By obstructing its interaction with DNA molecules, MGBLs block PARP-1 activity in vitro and in vivo, as we demonstrate using Drosophila, as well as human cancerderived cells. We also demonstrate synergistic inhibition of PARP-1, combining MGBLs with conventional NAD+-dependent inhibitors in human cancer cells. These results suggest that combining different classes of PARP-1 inhibitors can precisely modulate PARP-1 activity in living cells, thus holding promise for new avenues of cancer treatment.

\section{INTRODUCTION}

Poly ADP-ribose (ADPr) polymerase 1, PARP-1, is an essential protein involved in a wide range of cellular activities [1]. PARP-1 catalyzes the transfer of ADPr moiety onto protein acceptors or onto existing poly(ADPribose) chains by utilizing the glycolytic intermediate nicotinamide adenine dinucleotide (NAD+) as a source of ADPr [1-3]. Automodification of PARP-1 and its target proteins is accomplished by adding ADPr to glutamic residues $[1,2]$. The addition of ADPr polymers regulates the catalytic and DNA binding activity of PARP-1, as well as the cellular activity and localization of its target proteins. PARP-1 enzymatic activity is required for normal assembly of higher-order chromatin structures and the transcriptional activation of heat-shock-dependent, NFkB-dependent, ecdysteroid-dependent, and ribosomal genes [4-6]. In clinical studies, inhibitors of PARP-1 have been shown to selectively eliminate tumor cells [7-9]. Therefore, PARP-1 inhibitors have recently found widespread use in the development of novel strategies for cancer treatment, and several PARP-1 inhibitors are currently undergoing phase I/II trials for FDA approval for treatment of tumors $[10,11]$. However, a number of clinical studies have reported setbacks in research on PARP-1-based anticancer therapies [12,13]. Most PARP1 inhibitors have been designed to compete with NAD for a binding site on the PARP-1 molecule (Figure 1A). Since NAD is one of the most common cofactors involved in many eukaryotic pathways, this strategy resulted in the discovery of many nucleotide-like inhibitors that have a fairly low specificity to PARP-1 and also target other enzymatic pathways involving NAD and nucleotides as cofactors. Moreover, cancer cells tend to rapidly develop dynamic resistance against a single anticancer drug [14]. Thus, greater efficacy of PARP-1 inhibition could be 
attained by targeting multiple known routes of PARP-1 activation to develop combination therapies.

At least three points of PARP-1 protein control in vivo have been documented: 1) binding with NAD that serves as a substrate and a source of ADP-ribose for PARP-1 [1,2], 2) activation of PARP-1 via interaction with specific histones $[15,16]$, and 3 ) activation of PARP-1 by interaction with DNA [1,2] (Figure 1A). The two pathways of PARP-1 targeting to chromatin and regulation involve DNA-dependent and H4-dependent PARP-1 activations $[15,16]$. The first pathway controls PARP-1 binding to inactive chromatin and regulation via the interaction of Zn-finger of PARP-1 with the DNA molecule [17]. Consequently, small molecules that compete with PARP-1 for DNA binding might be a significant nexus of inhibition. In support of this notion, a recent study suggested that at least some PARP-1 inhibitory compounds do not compete with NAD and, therefore, may act by inhibiting DNA-binding [18-20]. Since a number of different small molecules, known as minor groove binding ligands (MGBL), can influence DNA-mediated enzymes [21], we tested several of them for their ability to inhibit PARP1 in vitro. Unlike intercalating DNA-binding molecules, which are extremely toxic and mutagenic [22,23], the minor groove binding small molecules from the Hoechst and diminazene groups do not induce point mutations or otherwise possess a very weak base-pair substitution potential [24-26]. As point mutagenesis is one of the main causes of drug resistance in cancer chemotherapy, nonmutagenic minor groove binding small molecules may show promise in future drug development [27]. Some of the compounds from this group are already in clinical use $[28,29]$. We demonstrated that these well-known minor groove binding small molecules may serve as potent PARP-1 inhibitors that target the DNA-dependent pathway of PARP-1 regulation. According to our findings, MGBLs prevent PARP-1 activation by competing for preferential binding sites on the DNA molecule. This mechanism of PARP-1 inhibition by MGBLs affords these molecules two possible types of application: as self-acting cytotoxic agents and as a component of combination chemotherapy preventing DNA repair by PARP-1, thereby facilitating DNA damage in cancer cells caused by other anticancer drugs [30].

\section{RESULTS}

\section{MGBLs inhibit PARP-1 by blocking its interaction with DNA}

We compared the effects of 6 different MGBLs on PARP-1 activation by DNA and by $\mathrm{H} 4$ in vitro. 4ANI, a NAD competitor, was used as a positive control for both types of PARP-1 activation. Ethidium bromide
(EtBr), representing a small molecule possessing high affinity to DNA, was also included in the analysis. We observed a strong and selective inhibitory effect for DAPI, diminazene, Hoechst $\mathrm{t}^{33342}$, Hoechst ${ }^{33258}$, and ethidium bromide, all of which blocked the DNA-dependent pathway of PARP-1 activation with remarkable specificity (Figure 1B). The more lipophilic Hoechst ${ }^{33342}$ exhibits significantly higher cell permeability than Hoechst ${ }^{33258}$ and possesses proapoptotic effect on cancer cells [30]. Therefore, we first tested Hoechst ${ }^{33342}$ to examine its ability to regulate PARP-1 functions in vitro. Different dilutions of Hoechst ${ }^{33342}$ had a gradual effect on DNAdependent PARP-1 inhibition, but no effect on H4dependent PARP-1 activity (Figure 1C-D). Preincubation with Hoechst ${ }^{33342}$ (Figure 1E-F) or diminazene (Figure 1F) completely disrupted the physical interaction of PARP-1 with DNA, but not with H4, thereby abolishing PARP-1 activation by DNA, but not by the histone (Figure 1F-H). Such specificity prompted us to test the effects of MGBLs on PARP-1 function in vivo.

\section{MGBLs compete with PARP-1 for DNA-binding}

Our data demonstrate that MGBLs specifically eliminate DNA-dependent functions of PARP-1. Moreover, our in vitro experiments showed that MGBLs inhibit PARP-1 by blocking the binding of PARP-1 to DNA molecules (Figure 1E,F), but not by directly interacting with PARP-1 or blocking PARP-1 interactions with other proteins (Figure 1E,F). To explore possible mechanisms of MGBL-mediated disruption of PARP1-DNA interaction, we superimposed two previously reported crystal structures of Hoechst ${ }^{33342}$-DNA [37] (PDB Code 129D) and PARP-1 Zn-finger-DNA [38,39] complex (PDB Code 4AV1) (Figure 2A,B,B' and S1). As Hoechst ${ }^{33342}$ is known to interact with the central AT base pairs $[30,40,41]$ in the duplex DNA, these base pairs were aligned and superimposed on the PARP Znf2 minor groove-interacting base pairs. As shown in Figure 2 and $\mathrm{S} 1$, binding of Hoechst ${ }^{3342}$ (magenta molecule) would preclude insertion of the key minor groove binding residue of Znf2, R122 (shown in green). Taken together, these data suggest that the presence of the minor groove binding dye would be expected to severely disrupt the binding of PARP-Zn fingers with DNA.

\section{The minor groove binding molecules Hoechst ${ }^{33342}$ and diminazene disrupt DNA-dependent PARP-1 localization and functions in vivo}

Unlike mammals with at least 17 PARPs, Drosophila has only one nuclear PARP, corresponding to human PARP-1 [4]. We recently demonstrated how DNA-dependent and histone-dependent functions of PARP-1 can be experimentally separated in Drosophila 
A
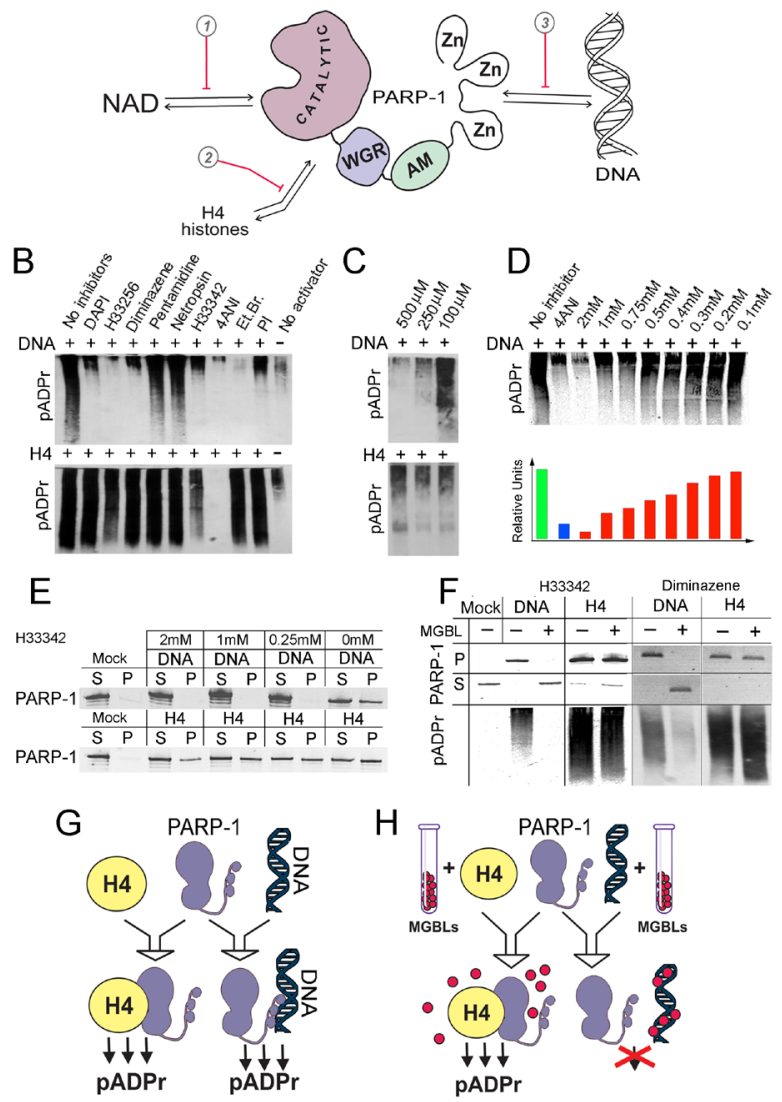

Figure 1: DNA-binding small molecules inhibit PARP-1 protein by blocking its interaction with DNA. A. Schema illustrates three ways of PARP-1 regulation: 1) competing with NAD binding, which represents the current design method; 2) disrupting PARP-1 interaction with histones; and 3) disrupting PARP-1 interaction with DNA. B. DNAbinding small molecules inhibit DNA-dependent, but not histone H4-dependent, PARP-1 activation. DNA (top) or H4 reaction mixtures were preincubated with different DNA-binding compounds or NAD competitor 4ANI, followed by mixing with PARP-1 and NAD. Accumulation of pADPr was detected on Western blots. C,D. Minor groove binding molecule Hoechst ${ }^{33342}$ specifically inhibits DNA-dependent PARP-1 activation (C). Panel D shows Western blot (top) and quantification (bottom) of amount of pADPr accumulation after PAPR-1 activation by DNA with and without NAD competitor 4-ANI or with dilution of Hoechst ${ }^{33342}$. E. Hoechst ${ }^{33342}$ disrupts PARP-1 binding to DNA (top), but not to histone H4 (bottom). Either DNA or histone $\mathrm{H} 4$ was covalently coupled to $\mathrm{CnBr}$ beads, pre-incubated with or without Hoechst ${ }^{33342}$, and incubated with PARP-1-containing solution. After precipitation of beads, pellet $(\mathrm{P})$ and solution (S) were subjected to PAGE and Western blot. The presence of PARP-1 in pellet and solution was detected on Western blot. F. MGBLs inhibit PARP-1 via disruption of PARP-1 interaction with DNA. The binding-activation assay is shown. Sepharose beads with covalently attached DNA or $\mathrm{H} 4$ were preincubated with $0 \mathrm{mM}$ or $0.25 \mathrm{mM}$ solution of Hoechst $\mathrm{t}^{33342}$ or diminazene, incubated with PARP-1, and mixed with NAD. The presence of PARP-1 and pADPr in pellet $(\mathrm{P})$ and solution $(\mathrm{S})$ was detected on Western blot after PAGE. G,H. Diagram illustrates how MGBLs hinder PARP-1 DNA-dependent activation by obstructing PARP-1 interaction with DNAs.
[17]. This makes the fruit fly invaluable in studying specific functions of PARP-1. We therefore examined PARP-1 inhibition by Hoechs ${ }^{33342}$ in fruit fly. Precise measurement of pADPr levels in the wild-type fruit fly is complicated by the abundance of PARG protein, which rapidly cleaves pADPr in vivo, as well as during protein extract preparation [42]. To accurately measure pADPr levels, we performed inhibitory assays in the absence of endogenous PARG using parg $^{27.1}$ mutant animals [42]. Asynchronous $\operatorname{parg}^{27.1}$ embryos and larvae were fed fruit fly food premixed with Hoechst ${ }^{33342}$ solution, and mature wandering third-instar larvae were collected after 16 or 39 hrs. When compared to wild-type animals at the same developmental stage, Parg mutant animals accumulated pADPr in a greater quantity (Figure $3 \mathrm{~A}$ ). However, culturing Drosophila in Hoechst-containing media significantly diminished the amount of pADPr detected (Figure 3A,B). Importantly, the agent approved in veterinary medicine, diminazene, showed a magnitude of PARP-1 inhibition in Drosophila similar to that of Hoechst (Figure S2A). The nucleoplasmic concentration of Hoechst and diminazene that was used during these experiments was significantly below saturation of their
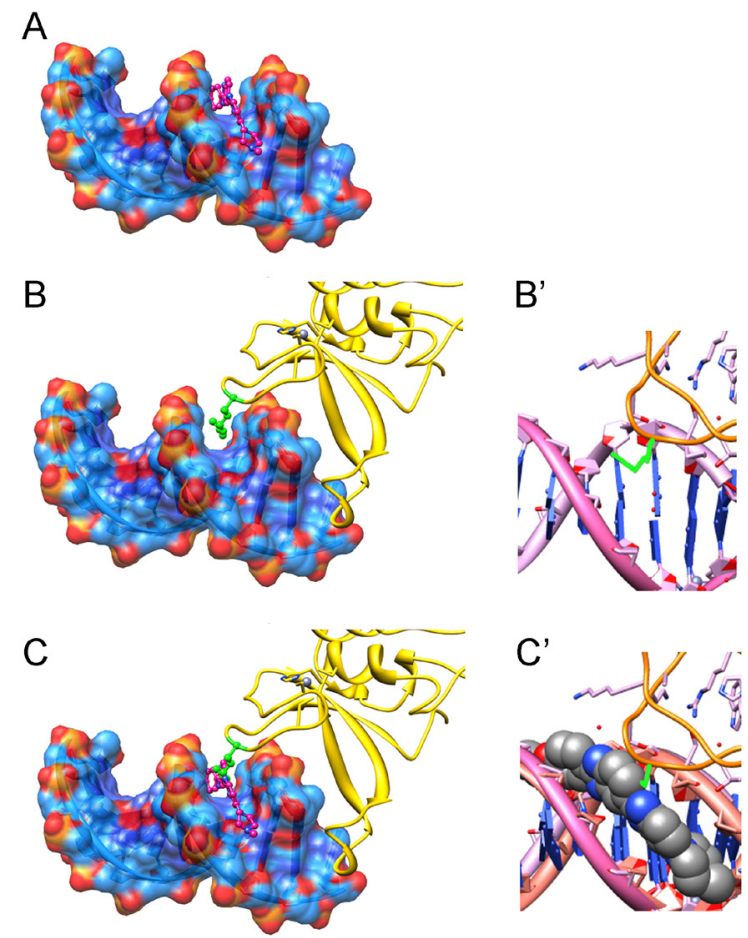

Figure 2: Model showing how PARP-1 protein competes with Hoechst ${ }^{33342}$ for DNA-binding (based on published crystallography data). The presence of MGBLs on DNA interferes with ZN-finger R122 intercalation between phosphor-sugar backbones in minor groove. Hoechst $\mathrm{t}^{33342}$ docked to the minor grove of DNA duplex (according to PDB Code 129D) (A) and PARP-1 Zn-finger docked to DNA complex (B, B') (according to PDB Code 4AV1). C, C' shows 45 A overlap between key Argenine 122 residue (green) of PARP-1 Zn-finger and Hoechst molecule. 
binding sites on DNA. Thus, these observations strongly suggest that MGBLs inhibit PARP-1 by competing with it for specific preferential binding sites on the DNA molecule, instead of nonspecifically obstructing PARP-1 binding to DNA by covering most of its length. These data confirm that MGBLs can function as potent PARP-1 inhibitors.

We next tested whether MGBLs could specifically affect DNA-dependent PARP-1 functions in fruit fly. Targeting of PARP-1 to the heterochromatin area of Drosophila genome and PARP-1-dependent transcriptional silencing are both controlled by the DNA-binding Zn-fingers of PARP-1 [17]. In wildtype Drosophila, PARP-1 has a broad, yet patterned, distribution along chromosomes, displaying considerable accumulation in regions of inactive, condensed chromatin
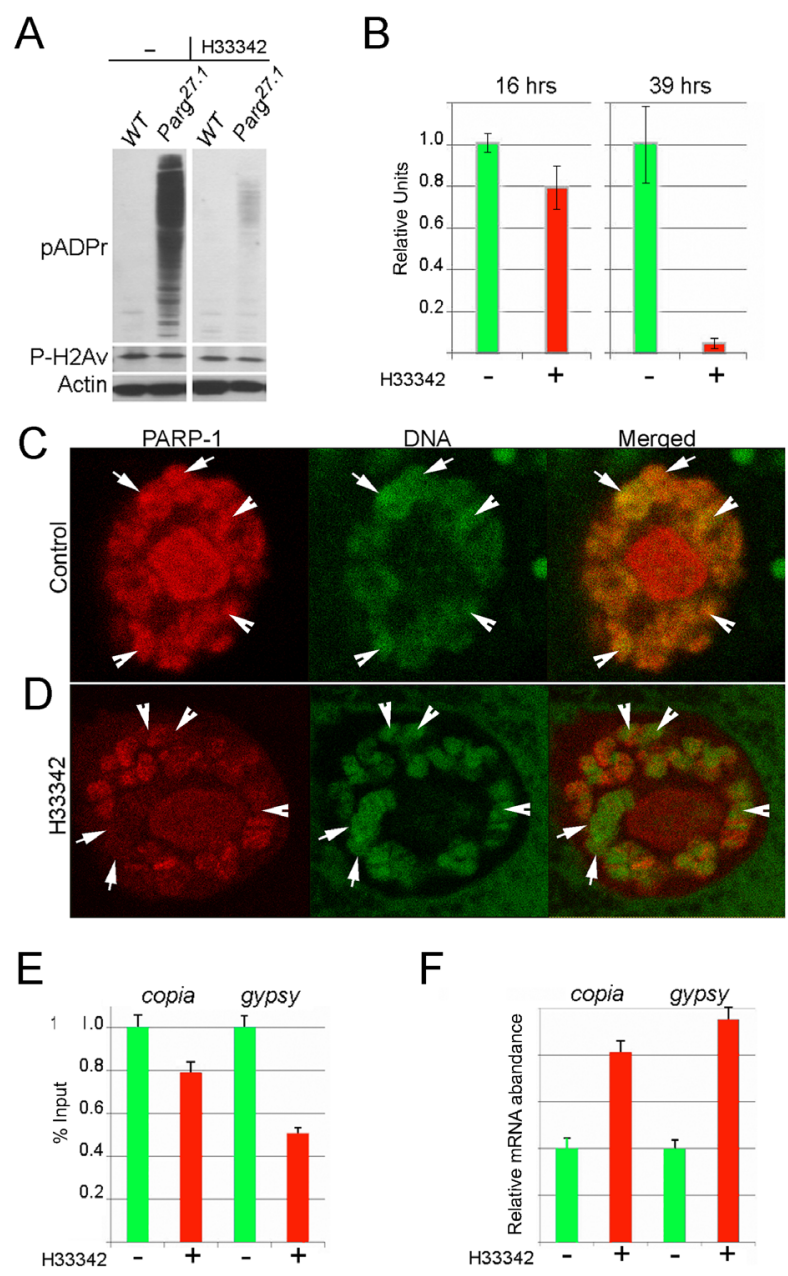

F
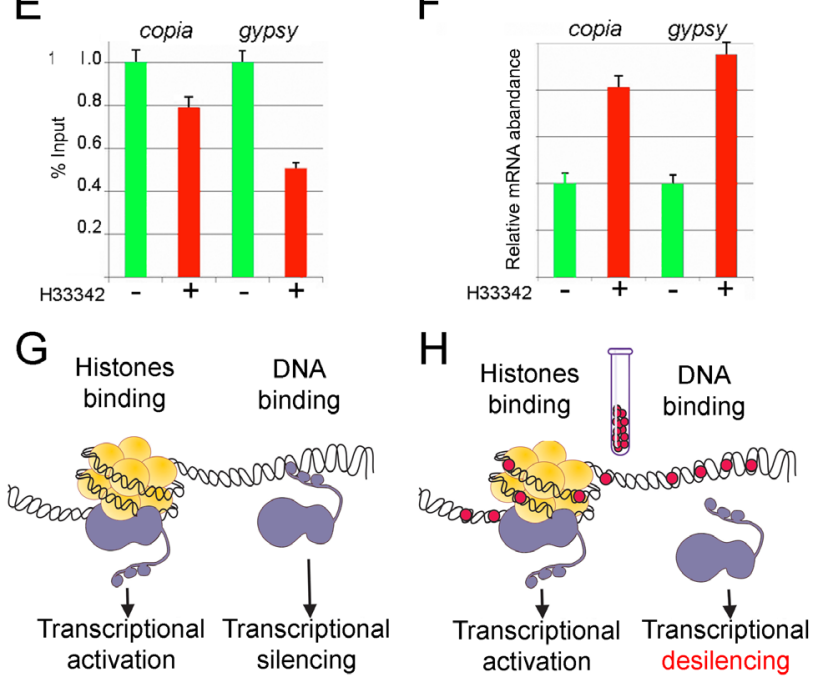

(heterochromatin) with high DNA content (Figure 3B). Culturing wild-type Drosophila with Hoechst ${ }^{33342}$ (Figure 3C,D) or diminazene (Figure S2B) eliminates PARP-1 protein accumulation almost completely from "dense" chromatin, which corresponds to constitutive heterochromatin and intercalary heterochromatin, but it does not affect the binding of PARP-1 in decondensed loci that have low DNA content (Figure 3C-D; S2B). This observation suggests that specific DNA-dependent PARP1 targeting to chromatin is inhibited by MGBLs.

We have previously shown that Zn-fingerdependent PARP-1 binding to heterochromatin is required for silencing of repeated heterochromatic DNAs [17]. Therefore, elimination of PARP-1 targeting to heterochromatin by Hoechst ${ }^{33342}$ should disrupt this silencing. We examined localization of PARP-1 in heterochromatin in Hoechst ${ }^{33342}$-treated animals and control using immunostaining of Drosophila polytene chromosomes and ChIP approaches. While PARP-1 in control animals shows significant accumulation on sequences of the retrotransposable elements copia and gypsy (typical content of silent chromatin), the amount of PARP-1 attached to these sequences is diminished after 39 hrs of Hoechst ${ }^{33342}$ treatment (Figure 3E). Moreover, we found that Hoechst ${ }^{33342}$-treated animals dramatically overproduce mRNA of both retrotransposons (Figure 3F). Thus, MGBLs appear to disrupt PARP-1 interaction with DNA in Drosophila and abolishes proper targeting

Figure 3: Minor groove binding molecule Hoechst ${ }^{33342}$ disrupts DNA-dependent PARP-1 localization and functions in vivo in Drosophila. A-B. A comparative analysis of PAPR-1 protein activity in the parg $^{27.1}$ mutant thirdinstar larvae cultured with or without Hoechst ${ }^{33342}$ in the media. To detect pADPr on Western blot, mAb $10 \mathrm{H}$ antibody against pADPr was used. pAb antibodies against phosphorylated $\mathrm{H} 2 \mathrm{Av}$ and Actin were used as a loading control. B. Quantification of pADPr accumulation in the parg $^{27.1}$ mutant third-instar larvae cultured with or without Hoechst ${ }^{33342}$ after 16 and 39 hrs of treatment. C-D. The treatment with Hoechst ${ }^{33342}$ disrupts Znfinger 1-dependent PARP-1 localization in heterochromatin of Drosophila. Comparison of PARP-1 protein localization in salivary gland polytene nuclei in untreated control (C) and after 39 hrs of culturing with Hoechst ${ }^{33342}$ (D). PARP-1 is shown in red color and DNA in green. Constitutive heterochromatin is outlined with arrows. Arrowheads point to intercalary heterochromatin. E. Compared to wild-type untreated animals, PARP-1 protein binds chromatin of the heterochromatic elements copia and gypsy significantly less after culturing with Hoechst $\mathrm{t}^{33342}$, as determined by ChIP assay. F. The quantitative RT-PCR assay shows that treatment with Hoechst ${ }^{33342}$ disrupts PARP-1-dependent silencing of the heterochromatic elements copia and gypsy. G,H. Diagram illustrating effects of MGBLs on DNA-dependent PARP-1 functions in vivo. G. In the absence of MGBLs, PARP-1 binding to DNA leads to transcription silencing, while PARP-1 interaction with nucleosomal histones leads to transcription activation. H. MGBLs obstruct PARP-1 binding to DNA, leading to desilencing of transcription; MGBLs have no effect on PARP-1 interaction with nucleosomal histones. 
of PARP-1 to heterochromatin, leading, in turn, to the desilencing of retrotransposable elements (Figure 3G,H).

\section{MGBLs synergistically interact with a classical PARP-1 inhibitor to block PARP-1 functions in human cancer-derived cells}

The efficacy of MGBL action on PARP-1 in vitro and in vivo suggests that MGBLs can be a starting point for developing novel drugs against PARP-1 to treat malignant tumors sensitive to PARP-1 inhibition. It has been shown that cells sensitive to PARP-1 inhibitors tend to have high preexisting levels of poly(ADP-ribose) [43]. Therefore, we tested Hoechst ${ }^{33342}$ using breast cancer-derived BT474 cells, which overaccumulate pADPr. Treatment of this cell culture with a NAD competitor, 4ANI (Figure 4A) or Olaparib (Figure 4B), diminishes pADPr amounts (Figure 4A). Similarly, Hoechst ${ }^{33342}$ (Figure 4A) and diminazene
(Figure 4B) treatments block pADPr accumulation, suggesting that MGBLs are effective inhibitors of PARP1 in human cells, just as in Drosophila.

To test the ability of DNA-dependent PARP1 inhibitors to work synergistically with the classical PARP-1 inhibitor based on the competition with NAD, we compared PARP-1 activity in BT474 cells following dual and monotherapies with each reagent. Considerable decrease in PARP-1 activity in BT474 cells confirms that dual treatment with Hoechst ${ }^{33342}$ and 4ANI (Figure 4C) and/or with diminazene - Olaparib (Figure 4D) inhibits PARP-1 activity with significantly greater efficacy than either of these inhibitors applied separately (Figure 4C-E). Our findings demonstrate (Figure 4D) that both MGBLs and NAD-competitors are cytotoxic to human cancerderived cells at very high concentrations only. Neither MGBLs nor NAD-competitors suppress the proliferation of cancer-derived cells, nor do they affect cell cycle progression (Supplemental Figures S3). Therefore, we
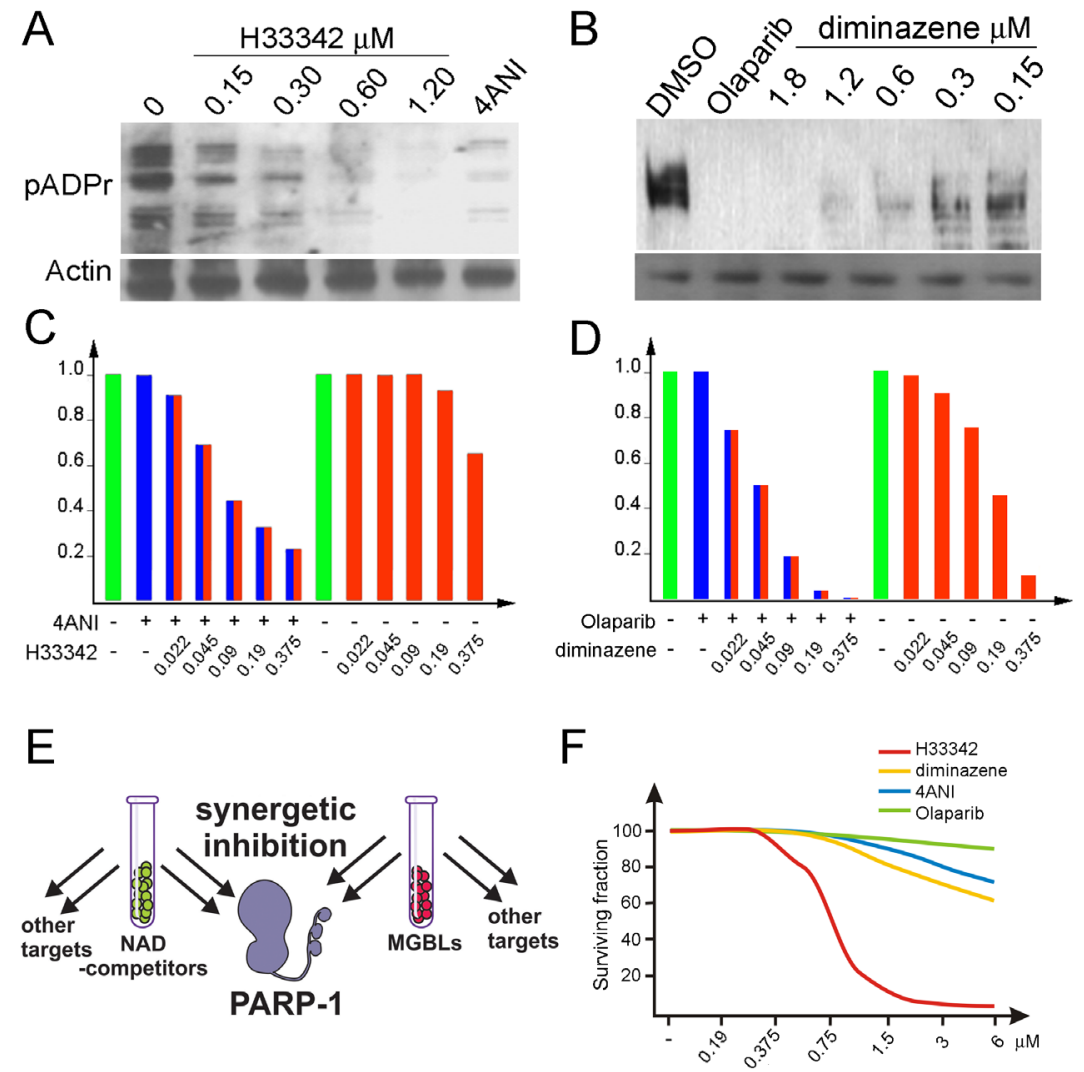

Figure 4: MGBLs inhibit PARP-1 activity in human cancer-derived cells. A-B. A comparative analysis of PARP-1 activity in BT474 cells cultured with and without 4ANI and Hoechst $t^{33342}$ (A) or Olaparib and diminazene (B). To detect pADPr on Western blot, mAb $10 \mathrm{H}$ antibody against $\mathrm{pADPr}$ was used. pAb antibodies against Actin were used as a loading control. C-D. MGBLs synergistically interact with classical PARP-1 inhibitors to block PARP-1 functions in vivo in human cancer cells. (C) Quantification of pADPr amounts in BT474 cells treated with 4ANI and Hoechst ${ }^{33342}$ separately, as well as with combinations of 4ANI and different concentrations of Hoechst ${ }^{33342}$. (D) Quantification of pADPr amounts in BT474 cells treated with Olaparib and diminazene separately, as well as with combinations of Olaparib and different concentrations of diminazene. The amounts of poly(ADP-ribose), which reflect PARP-1 enzymatic activity, were detected after PAGE on Western blot using the anti-pADPr antibody and were quantified independently, using the Image Quant Software Package. E. Diagram illustrating the additive effect of MGBLs and NAD-competitors acting together. Apart from PARP-1, each of these chemicals targets other pathways. However, acting together, they are able to hinder PARP-1 activation via both routes with great efficacy. F. A cell

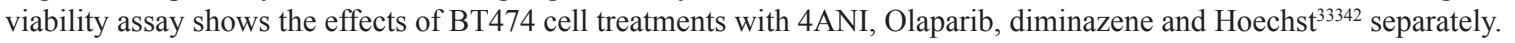


further tested the ability of MGBLs and NAD-competitors to specifically suppress the tumorigenic potential of human cancer cells. For these experiments, we used the classical PARP-1 inhibitor Olaparib and the MGBL diminazene, both effectively inhibit PARP-1 in vivo at nanomolar concentrations.

We compared the capacity of these small molecules to suppress the clonogenic potential of human cancerderived cells. To accomplish this, we preselected cancer-derived cell lines [31-35], which, unlike normal human cells, demonstrate severe misregulation of
pADPr pathway: BT474 (breast cancer), PC3 (prostate cancer), Skov3 and Ovca432 (ovarian cancer), as well as NKE and PNX (renal cell carcinoma (RCC) (Figure 5A). Cancer-derived cells express an abnormally high level of the PARP-1 protein and no PARG (Figure 5A). To examine the ability of diminazene and Olaparib to suppress the clonogenic potential [36] of human cancerderived cells, we treated cells with these compounds separately and together. Although individual effects of Olaparib and diminazene vary from one cancer cell line to another, dual treatment with Olaparib and diminazene
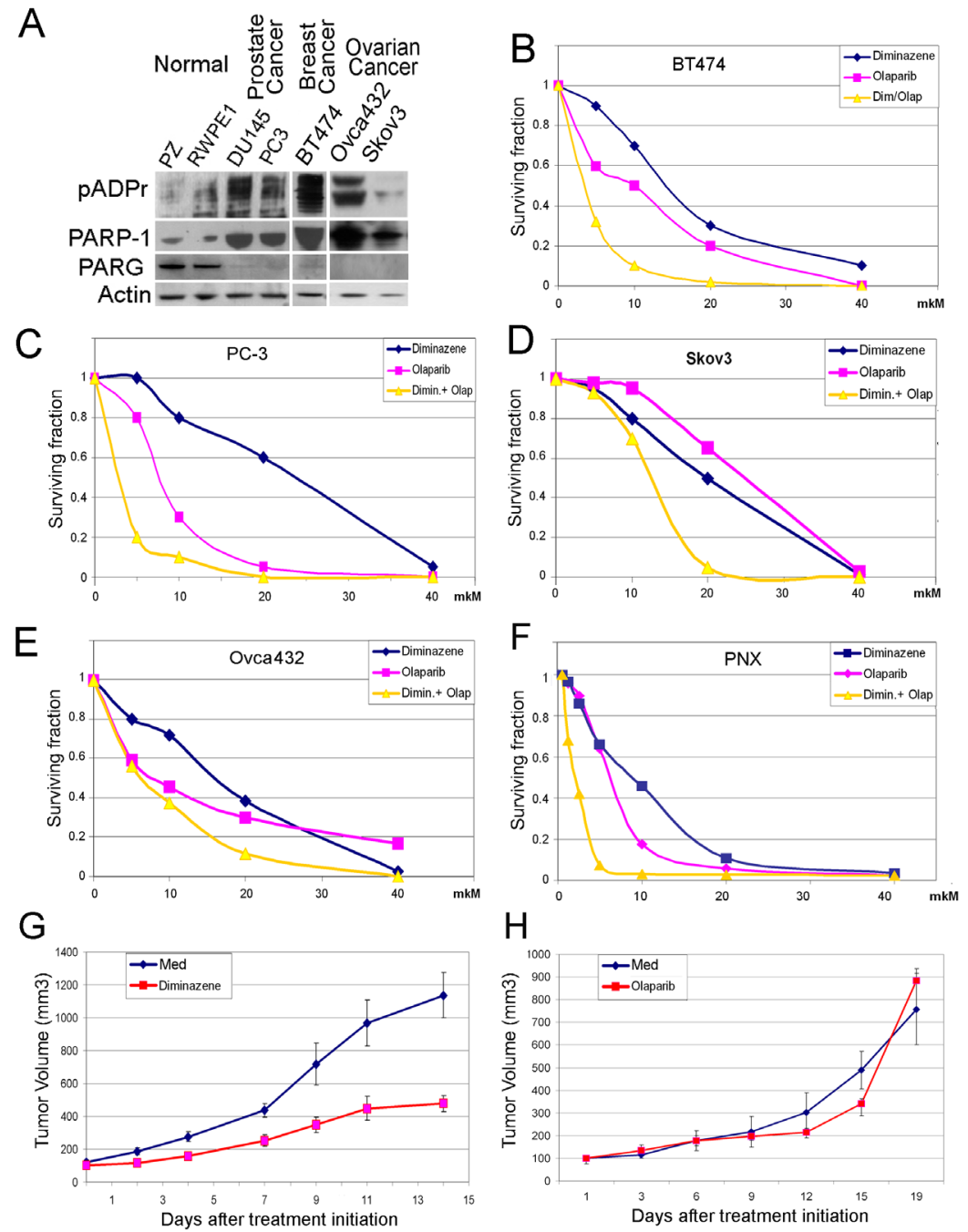

Figure 5: MGBLs suppress the growth of cancer cells. Malignancy arising from cancer-derived cells is associated with aberrations in the regulation of pADPr turnover. Equal amounts of total protein extracts from normal (RWPE1 and PZ) and cancer-derived cells, including BT474 (breast cancer), DU145 and PC3 (prostate cancer), Skov3 and Ovca432 (ovarian cancer), were analyzed after PAGE on Western blots using anti-pADPr, anti-PARG, anti-PARP-1, and anti-Actin antibodies. Both cancer cell lines show defects in PARG expression and overproduction of PARP-1 and pADPr. B-F. Clonogenic Cell Survival Assay: BT474 (breast cancer) (B), PC-3 (prostate cancer) (C) Skov3 and Ovca432 (ovarian cancer) (D,E), PNX (renal cell carcinoma (RCC)). (F) Cells were plated into 24-well plates, allowed to adhere overnight and treated with increasing concentrations of diminazene (blue), Olaparib (magenta) and both (yellow) for 14 days. Colonies were counted and plotted on the graph. G-H. Diminazene inhibitor suppresses growth of patient-derived RCC xenograft tumors in vivo. Ectopic RCC xenograft tumors were established in 6-week-old male C.B17/Icr-scid mice using patient-derived PNX tumor cells. Animals were treated intraperitoneally with the MGBL diminazene (23 mg/kg) or vehicle $(0.9 \% \mathrm{NaCl})(\mathrm{G})$, classical PARP-1 inhibitor Olaparib (Olap) $(50 \mathrm{mg} / \mathrm{kg}$ ) or vehicle (PBS + 10\% (2-Hydroxypropyl)-b-cyclodextrin) (H) 5 days a week for 17 days (G-H). Values shown represent means $(\mathrm{n}=5)+$ SEM. 
consistently demonstrated prominent synergistic efficacy in eliminating cancer-derived clones (Figure 5B-F). In light of encouraging in vitro data, we next examined the antitumor activity of non-NAD-like inhibitors using a renal cell carcinoma ( $\mathrm{RCC}$ ) xenograft tumor established from patient-derived tumor cells. As demonstrated in Figure 5G-H, animals treated with the MGLB diminazene showed a significant inhibition of tumor growth relative to control animals and animals treated with the classical PARP-1 inhibitor Olaparib. Importantly, treatment with diminazene was well tolerated by all animals, with no apparent signs of toxicity. These results are promising for the use of DNA-dependent PARP-1 inhibitors in therapeutic applications, when combined with conventional NAD-competing agents.

\section{DISCUSSION}

By demonstrating an inhibitory effect of MGBLs on PARP-1, an enzyme required for DNA repair and, by extension, cell survival, we have explained the cytotoxicity of this compound that has been observed in a number of cell lines in vitro and in xenografts $[27,44]$ (Figure 5G$\mathrm{H})$. According to our findings, MGBLs prevent PARP-1 activation by competing for preferential binding sites on the DNA molecule. This mechanism of PARP-1 inhibition by MGBLs affords these molecules two possible types of application: as self-acting cytotoxic agents and as a component of combination chemotherapy to prevent DNA repair by PARP-1, thereby facilitating DNA damage in cancer cells caused by other anticancer drugs $[30,45,46]$.

Previously, MGBLs were shown to influence the activities of many DNA-processing proteins, such as topoisomerases, helicase, TATA box binding protein, replication protein A and others [30]. Most proteins that bind specifically to AT-rich DNA regions are considered to have extensive contact within the minor groove. Therefore, their inhibition should be mediated by direct steric hindrance $[45,46]$. Each MGBL binds AT-rich DNA in a sequence-specific manner, inhibiting a unique suite of DNA-binding proteins, thereby altering the pattern of protein activity and gene expression. Protein activity can be altered by at least two mechanisms: inhibition of specific genes and direct steric hindrance between MGBLs and DNA-binding proteins for specific binding sites on AT-rich DNA regions. An alternative mechanism of action by Hoechst ${ }^{33342}$ could involve changes to bent DNA conformations in genomic DNA. Since it has been suggested that 1) PARP-1 Zn-finger may preferentially interact with bent DNA [39] and 2) Hoechst ${ }^{33342}$ has been shown to mediate DNA bending $[47,48]$, it seems plausible that an alternate mechanism of PARP-1 inhibition by Hoechst $^{33342}$ could involve changes in the degree of DNA bending.

Because cancer cells multiply rapidly, any disruption of the household gene expression caused by
MGBLs affects them more rapidly and to a greater extent than normal cells. As with other drugs, antitumor action of MGBLs is expected to be restricted by their toxicity to normal cells, in particular to hematopoiesis and the intestinal epithelium. However, unlike other drugs, MGBLs produce a unique profile of gene expression inhibition and protein synthesis that is specific for each MGBL. Therefore, MGBL-based drugs have a potential for selectivity against tumors in which mutations have established a distinct profile of household gene activity. Such MGBL-based drugs would have superior specificity and efficacy against sensitive tumorigenic cells, as suggested by other studies [40,41].

Taken together, the findings of this study lay the groundwork for the development of new small molecules directed against PARP-1/DNA activity, either as a monotherapy or in combination with known NADcompeting compounds or cytotoxic chemotherapies. New efficient and specific strategies for treating human malignancies based on PARP-1 inhibition will likely be developed in the future. More importantly, this study introduces a novel approach to designing PARP-1 inhibitors and proposes new strategies for eliminating tumorigenic cells and overcoming resistance to the NADcompetitive class of inhibitors.

\section{MATERIALS AND METHODS}

\section{Human cell cultures}

Human breast carcinoma cell line BT474 [31] was cultured in RPMI 1640 with $10 \%$ FBS, sodium pyruvate $(10 \mathrm{mM}), \mathrm{N}-2$-hydroxyethylpiperazine-N'-2ethanesulfonic acid (10mM) and antibiotics. Androgenindependent human PC-3 prostate cancer cells [32] were obtained from ATCC (Rockville, MD). Cells were cultured in RPMI 1640 (Bio-Whittaker, Walkersville, MD) supplemented with 10\% FBS (Hyclone, Logan, UT), penicillin $(100 \mathrm{U} / \mathrm{ml})$, streptomycin $(100 \mathrm{ug} / \mathrm{ml})$, sodium pyruvate $(1 \mathrm{mM})$ and non-essential amino acids $(0.1 \mathrm{mM})$ under conditions indicated in the figure legends. Normal prostate epithelium cells RWPE-1 [33] were obtained from ATCC (Rockville, MD). RWPE-1 cells were maintained in Keratinocyte-Serum Free medium (Invitrogen, Carlsbad, CA) supplemented with $5 \mathrm{ng} / \mathrm{ml}$ of human recombinant EGF and $0.05 \mathrm{mg} / \mathrm{ml}$ of bovine pituitary extract. Ovarian cancer cell lines [34] were a kind gift from the Dennis Connolly lab. The NKE cells [35] were obtained from ATCC (Rockville, MD). The PNX cell line was a kind gift from Dr. Igor Astsaturov, MD, PhD (Fox Chase Cancer Center, Philadelphia, PA). Tumor cells were isolated from tumor tissue specimen obtained with written informed consent and Fox Chase Cancer Center Institutional Review Board approval (IRB approved protocol \#12-822) from 
a patient undergoing tumor resection at the Fox Chase Cancer Center.

\section{PARP-1 inhibitory assay in human cell culture}

Different doses of Hoechst $\mathrm{t}^{33342}$, diminazene and Olaparib were added to the cells cultured in the complete medium. After 24 or $48 \mathrm{hrs}$, cells were lysed, and protein samples were analyzed with SDS-PAGE and Western Blot using anti-pADPr antibody.

\section{Treatment of Drosophila with MGBL PARP-1 inhibitors}

WT flies were mass mated, and 10 females and 5 males were placed in vials with standard medium containing different doses of Hoechst (dose H10 - $330 \mu \mathrm{l}$ of $10 \mathrm{mM}$ Hoechst ${ }^{3342}$ per each $8.25 \mathrm{~g}$ of medium; dose $\mathrm{H} 20-330 \mu \mathrm{l}$ of $20 \mathrm{mM}$ Hoechst ${ }^{33342}$ per each $8.25 \mathrm{~g}$ of medium) or distilled water. After $24 \mathrm{hrs}$, the parents were removed. Each experimental and control group consisted of four vials. In each vial, the number of pupae and imagoes were calculated and compared among the groups using Student's $t$-test.

\section{Clonogenic Cell Survival Assay [36]}

Cells were plated into 24-well plates at a density of 2000 cells/well. Cells were allowed to adhere overnight at $37^{\circ} \mathrm{C}$ and treated with increasing concentrations of diminazene and Olaparib for 14 days. Colonies were fixed with $70 \%$ ethanol for $10 \mathrm{~min}$ and stained with $0.25 \%$ methylene blue in $30 \%$ ethanol for $10 \mathrm{~min}$. After that, staining solution was removed, and plates were rinsed with water. Colonies consisting of 50 cells or more were counted. Plating efficiencies (PE) were calculated as follows: $\mathrm{PE}=$ number of colonies/number of cells seeded. The surviving fraction (SF) was calculated as follows: SF $=$ number of colonies/number of cells seeded $\times \mathrm{PE}$.

\section{Ethics statement}

This study was carried out in strict accordance with the recommendations from the Guide for the Care and Use of Laboratory Animals, as provided by the American Association of Accreditation of Laboratory Animal Care (AAALAC).

\section{Statistics}

All data are presented as mean \pm SEM. Statistical analyses were done using 2-tailed Student's $t$-test. A P value of 0.05 or less was considered significant.

\section{ACKNOWLEDGMENTS}

We thank Drs. M. Robinson, I. Astsaturov and D. Connolly for providing human cancer-derived cells. Drs. D. Connolly, J. Peterson, M. Robinson, K. Pechenkina and David Martin provided comments on the manuscript. The molecular modeling analysis was performed in the FCCC Molecular Modeling Facility by Mark Andrake. The research was supported by grants from the National Institutes of Health (R01 GM077452 and R01 DK082623) to A.V.T. and from the Russian Foundation for Basic Research (\#13-04-01707-a, \#11-04-01978-a) and The European Association for Cancer Research (Travel Grant) to K.K.

\section{AUTHOR CONTRIBUTIONS}

Conceived and designed the experiments: KIK, EL, MGY, VK, AVT. Performed the experiments: KIK, EK, PM, KG. Analyzed the data: KIK, EK, PM, KG, AVT. Contributed reagents/materials/analysis tools: KIK, EK, VK, KG, AVT. Wrote the paper: KIK, AVT.

\section{Editorial note}

This paper acceptance was based in part on peerreview conduced by another journal, revisions and further internal peer-review by Oncotarget.

\section{REFERENCES}

1. Shall S, de Murcia G. Poly(ADP-ribose) polymerase-1: what have we learned from the deficient mouse model? Mutat Res. 2000; 460: 1-15.

2. D'Amours D, Desnoyers S, D'Silva I, Poirier GG. Poly(ADP-ribosyl)ation reactions in the regulation of nuclear functions. Biochem. 1999; J 342: 249-268.

3. Satos MS, Lindahl T. Role of poly(ADP-ribose) formation in DNA repair. Nature. 1992; 356: 356-358.

4. Tulin A, Stewart D, Spradling AC. The Drosophila heterochromatic gene encoding poly(ADP-ribose) polymerase (PARP) is required to modulate chromatin structure during development. Genes Dev. 2002; 16: 21082119.

5. Beneke S. Regulation of chromatin structure by poly(ADP)ribosylation. Front Genet. 2012; 3: 169.

6. Dantzer F, Amé JC, Schreiber V, Nakamura J, Ménissier de Murcia J. Poly(ADP-ribose) polymerase-1 activation during DNA damage and repair. Methods Enzymol. 2006; 409: 493-510.

7. Curtin NJ. PARP inhibitors for cancer therapy. Expert Rev Mol Med. 2005; 7: 1-20.

8. Virág L. Structure and function of poly(ADP-ribose) polymerase-1: role in oxidative stress-related pathologies. 
Curr Vasc Pharmacol. 2005; 3: 209-214.

9. Hiller D, Chu QD. Current Status of Poly(ADP-ribose) Polymerase Inhibitors as Novel Therapeutic Agents for Triple-Negative Breast Cancer. Int J Breast Cancer. 2012: 829315.

10. Davar D, Beumer JH, Hamieh L, Tawbi H Role of PARP Inhibitors in Cancer Biology and Therapy Curr Med Chem. 2012; 19: 3907-3921.

11. Annunziata C, Shaughnessy J. PARP as a novel therapeutic target in cancer. Clin Cancer Res. 2010; 16: 4517-4526.

12. Sandhu SK, Papadopoulos K, Fong PC, Patnaik A, Messiou C, Olmos D, Wang G, Tromp BJ, Puchalski TA, Balkwill F, Berns B, Seetharam S, de Bono JS, Tolcher AW. Firstin-human trial of a poly (ADP-ribose) polymerase (PARP) inhibitor MK-4827 in advanced cancer patients (pts) with antitumor activity in BRCA-deficient and sporadic ovarian cancers J Clin Oncol. 2010; 28: 233.

15. Pinnola A, Naumova N, Shah M, Tulin AV. Nucleosomal core histones mediate dynamic regulation of poly(ADPribose) polymerase 1 protein binding to chromatin and induction of its enzymatic activity. J Biol Chem. 2007; 282: 32511-32519.

13. Kummar S, Chen A, Parchment RE, Kinders RJ, Ji J, Tomaszewski JE, Doroshow JH. Advances in using PARP inhibitors to treat cancer. BMC Med. 2012; 10:25.

14. Mandery K, Glaeser H, Fromm MF. Interaction of innovative small molecule drugs used for cancer therapy with drug transporters. Br J Pharmacol. 2012; 165: 345-362

16. Kotova E, Pinnola AD, Tulin AV. Small-molecule collection and high-throughput colorimetric assay to identify PARP-1 inhibitors. Methods Mol Biol. 2011; 780:491-516.

17. Kotova E, Jarnik M, Tulin AV. Uncoupling of the transactivation and transrepression functions of PARP-1 protein. Proc Natl Acad Sci USA. 2010; 107: 6406-6411.

18. Ellisen LW. PARP inhibitors in cancer therapy: promise, progress, and puzzles Cancer Cell 2011; 19: 165-167.

19. Ferraris DV Evolution of poly(ADP-ribose) polymerase-1 (PARP-1) inhibitors. J Med Chem. 2010; 53: 4561-4584

20. Ma W, Halweg CJ, Menendez D, Resnick MA. Differential effects of poly(ADP-ribose) polymerase inhibition on DNA break repair in human cells are revealed with Epstein-Barr virus. Proc Natl Acad Sci USA. 2012; 109: 6590-6595.

21. Chen AY, Chiang Y, Gatto B, Liu LF DNA minor groovebinding ligands: A different class of mammalian DNA topoisomerase I inhibitors. Proc. Natl. Acad. Sci. USA. 1993; 90: 8131-8135.

22. Yielding LW, Brown BR, Graves DE, Yielding KL. Ethidium bromide enhancement of frameshift mutagenesis caused by photoactivatable ethidiumanalogs. Mutat Res. 1979; 63: 225-232.

23. Kirsanov KI, Lesovaya EA, Yakubovskaya MG, Belitsky GA. SYBR Gold and SYBR Green II are not mutagenic in the Ames test. Mutat Res. 2010; 699: 1-4.
24. Ferguson LR, Denny WA. Microbial mutagenic effects of the DNA minor groove binder pibenzimol (Hoechst 33258) and a series of mustard analogues. Mutat Res. 1995; 329: 19-27.

25. Wu XC, Marcinkowski K, Turner PM, Ferguson LR. Mutations induced by some DNA minor groove binding alkylators in AS52 Chinese hamster cells. Mutat Res. 2000; 448: 35-45.

26. Kakasi B, Kovats N, Nagy S. Assessment of the genotoxic potentialof Hoechst 33342, SYBR-14 and PI using the SOS ChromoTest. Biotech Histochem. 2012; 87: 372-375.

27. Baraldi PG, Boverz A, Fruttarolo F, Preti D, Tabrizi MA, Pavani MG, Romagnoli, R. DNA minor groove binders as potential antitumor and antimicrobial agents. Med Res Rev. 2004; 24: 475-528.

28. Bielawski K, Bielawska A, Popławska B, Surazyński A, Czarnomysy R. The effect of a novel dinuclear platinum complex with berenil and 2-picoline ligands on growth of human breast cancer cells. Acta Pol Pharm. 2010; 67: 609614.

29. Khan GS, Shah A, Zia-Ur-Rehman, Barker D. Chemistry of DNA minor groove binding agents. J Photochem Photobiol B. 2012; 115: 105-118.

30. Zhang X, Zhang SC, Sun D, Hu J, Wali A, Pass H, Fernandez-Madrid F, Harbut MR, Tang N New insight into the molecular mechanisms of the biological effects of DNA minor groove binders. PLoS One. 2011; 6(10).

31. Lasfargues EY, et al. Isolation of two human tumor epithelial cell lines from solid breast carcinomas. J. Natl. Cancer Inst. 1978; 61: 967-978.

32. Teper E, Makhov P, Golovine K, Canter DJ, Myers CB, Kutikov A, Sterious SN, Uzzo RG, Kolenko VM. The effect of 5-aminolevulinic acid and its derivatives on protoporphyrin IX accumulation and apoptotic cell death in castrate-resistant prostate cancer cells. Urology. 2012; 80(6): 1391.e1-7.

33. Makhov PB, Golovine KV, Kutikov A, Canter DJ, Rybko VA, Roshchin DA, Matveev VB, Uzzo RG, Kolenko VM. Reversal of epigenetic silencing of AP-2alpha results in increased zinc uptake in DU-145 and LNCaP prostate cancer cells. Carcinogenesis. 2011; 32(12): 1773-1781.

34. Berkowitz R, Kabawat S, Lazarus H, Colvin R, Knapp R, Bast RC Jr. Comparison of a rabbit heteroantiserum and a murine monoclonal antibody raised against a human epithelial ovarian carcinoma cell line. Am J Obstet Gynecol. 1983; 146(6): 607-612.

35. Biswas K, Richmond A, Rayman P, Biswas S, Thornton M, Sa G, Das T, Zhang R, Chahlavi A, Tannenbaum CS, Novick A, Bukowski R, Finke JH. GM2 expression in renal cell carcinoma: potential role in tumor-induced T-cell dysfunction. Cancer Res. 2006; 66(13): 6816-6825.

36. Mori S, Chang JT, Andrechek ER, Matsumura N, Baba T, Yao G, Kim JW, Gatza M, Murphy S, Nevins JR. Anchorage-independent cell growth signature identifies 
tumors with metastatic potential. Oncogene. 2009; 28(31): 2796-2805.

37. Sriram M, van der Marel GA, Roelen HL, van Boom JH, Wang AH. Conformation of B-DNA containing O6ethyl-G-C base pairs stabilized by minor groove binding drugs: molecular structure of d(CGC[e6G]AATTCGCG complexed with Hoechst 33258 or Hoechst 33342. EMBO J. 1992; 11: 225-232.

38. Eustermann S, Videler H, Yang JC, Cole PT, Gruszka D, Veprintsev D, Neuhaus D. The DNA-Binding Domain of Human PARP-1 Interacts with DNA Single-Strand Breaks as a Monomer through Its Second Zinc Finger. J Mol Biol. 2011; 407: 149-141.

39. Langelier M-F, Planck JL., Roy S, Pascal JM. Crystal Structures of Poly(ADP-ribose) Polymerase-1 (PARP1) Zinc Fingers Bound to DNA: structural and functional insights into DNA-dependent parp-1 activity. J. Biol. Chem. 2011; 286: 10690-10701.

40. Dervan PB. Molecular recognition of DNA by small molecules. Bioorg Med Chem. 2001; 9: 2215-2235.

41. Neidle S. DNA minor-groove recognition by small molecules. Nat Prod Rep. 2001; 18: 291-309

42. Tulin A, Naumova N, Menon A, Spradling AC. Drosophila Poly(ADP-Ribose) Glycohydrolase Mediates Chromatin Structure and SIR2-Dependent Silencing. Genetics. 2006; 172: 363-371.

43. Gottipati P, Vischioni B, Schultz N, Solomons J, Bryant HE, Djureinovic T, Issaeva N, Sleeth K, Sharma RA, Helleday T. Poly(ADP-Ribose) Polymerase is Hyperactivated in Homologous Recombination-Defective Cells. Cancer Res. 2010; 70: 5389-5398.

44. Mann J, Baron A, Opoku-Boahen Y, Johansson E, Parkinson G, Kelland LR, Neidle S. A New Class of Symmetric Bisbenzimidazole-Based DNA Minor GrooveBinding Agents Showing Antitumor Activity. J Med Chem. 2001; 44: 138-144.

45. Turner PR, Denny WA. The genome as a drug target: sequence specific minor groove binding ligands. Curr Drug Targets. 2000; 1: 1-14.

46. Tse WC, Boger DL. Sequence-Selective DNA Recognition: Natural Products and Nature's Lessons. Chem \& Bio. 2004; 11: 1607-1617.

47. Doshi P, Kaushal S, Benyajati C, Wu CI. Molecular analysis of the responder satellite DNA in Drosophila melanogaster: DNA bending, nucleosome structure, and Rsp-binding proteins. Mol Biol Evol. 1991; 8: 721-741.

48. Tawar U, Jain AK, Chandra R, Singh Y, Dwarakanath BS, Chaudhury NK, Good L, Tandon V. Minor groove binding DNA ligands with expanded A/T sequence length recognition, selective binding to bent DNA regions and enhanced fluorescent properties. Biochemistry. 2003; 42: 13339-13346. 